\title{
Kernos
}

Revue internationale et pluridisciplinaire de religion grecque antique

$20 \mid 2007$

Varia

\section{VALDÉs Miriam, El papel de Afrodita en el alto arcaísmo griego. Política, guerra, matrimonio e iniciación}

\section{Gabriella Pironti}

\section{(2) OpenEdition \\ Journals}

\section{Édition électronique}

URL : https://journals.openedition.org/kernos/347

DOI : $10.4000 /$ kernos.347

ISSN : 2034-7871

\section{Éditeur}

Centre international d'étude de la religion grecque antique

\section{Édition imprimée}

Date de publication : 1 janvier 2007

Pagination : 414-416

ISSN : 0776-3824

\section{Référence électronique}

Gabriella Pironti, « vaLdés Miriam, El papel de Afrodita en el alto arcaísmo griego. Política, guerra, matrimonio e iniciación », Kernos [En ligne], 20 | 2007, mis en ligne le 18 juin 2011, consulté le 08 septembre 2022. URL : http://journals.openedition.org/kernos/347 ; DOI : https://doi.org/10.4000/ kernos.347 
VALDÉs Miriam, El papel de Afrodita en el alto arcaísmo griego. Política, guerra, matrimonio e iniciación, Messina, Di.Sc.A.M., 2005. 1 vol. $18 \times 25 \mathrm{~cm}, 162$ p. (Polifemo Supplemento, 2). ISBN : 88-8268-014-2.

Au cours des dernières années, Aphrodite a fait l'objet de plusieurs études ${ }^{1}$. Témoignant également de cette attention renouvelée, le livre de Miriam Valdès a le mérite de prendre en considération les liens d'Aphrodite avec la sphère politique, un aspect encore largement négligé jusqu'à la moitié du siècle dernier, et de prêter attention aux cultes de l'Aphrodite armée ${ }^{2}$. Comme le titre de l'ouvrage l'indique explicitement, l'A. choisit un cadre chronologique bien précis, la haute époque archaïque, et c'est en rapport avec le processus de formation de la polis qu'elle essaie d'élucider les prérogatives d'Aphrodite dans plusieurs sphères à la fois, à savoir la politique, la guerre, le mariage et l'initiation. Il s'agit d'un projet ambitieux qui vise à écrire l'histoire même d'Aphrodite, dès son origine, à travers les phases de formation et d'expansion de son culte, jusqu'à l'intégration de la déesse dans les panthéons des cités grecques.

Une introduction analytique, qui tient aussi lieu de conclusion, anticipe largement les hypothèses et les arguments qui vont faire l'objet de six chapitres. Une attention particulière est réservée à la question des origines d'Aphrodite (Chap. I) : M.V. reprend la thèse bien connue qui fait d'Aphrodite l'héritière d'une déesse née dans l'île de Chypre de la rencontre entre la divinité locale et les divinités proche-orientales liées à la fois à la guerre et à la sexualité, sans exclure pour autant une composante mycénienne et une influence crétoise. L'A. élabore ensuite une hypothèse sur l'expansion du culte d'Aphrodite : lors d'une première phase d'expansion, que M.V. croit pouvoir dater du début de l'âge du fer, la déesse serait arrivée de Chypre en Grèce centrale avec ses prérogatives caractéristiques - l'amour et la


Phéniciens auraient joué un rôle important, et c'est à ce moment qu'arriverait en Grèce une Aphrodite caractérisée aussi par des traits guerriers. La poésie épique témoignerait de la difficile intégration au panthéon grec d'une déesse liée à la fois à la sexualité et à la guerre, les Grecs ayant accordé, dans ce domaine, leur préférence à une déesse vierge comme Athéna.

M.V. ne se contente pas de retracer l'origine d'Aphrodite, mais essaie de suivre l'évolution de sa personnalité, en la replaçant à l'intérieur des processus sociaux qui aboutissent à la formation de la cité grecque. Elle est convaincue que les traits guerriers d'Aphrodite, avant de perdre progressivement leur signification, ont été accueillis dans la société grecque, et cela au moment de la constitution d'une puissante aristocratie guerrière. Selon M.V., cette déesse représentait la candidate adéquate pour présider aux initiations parallèles des jeunes gens au mariage et à la guerre, et aux travestissements rituels censés les accompagner. De même, sur la base de certains rituels où serait à l'œuvre un schéma d'inversion de rôles, visant au renforcement de l'ordre établi, M.V. retrouve dans les anciennes images de l'Aphrodite guerrière la trace du processus de formation de la cité au moment où elle s'affirme, par

${ }^{1}$ La publication, en 1994, de l'ouvrage de V. Pirenne-Delforge sur les cultes de la déesse a sans doute contribué à ce renouveau d'intérêt: L'Aphrodite grecque. Contribution à l'étude de ses cultes et de sa personnalité dans le panthéon archaïque et classique, Athènes-Liège, 1994 (Kernos, suppl. 4). Voir, par exemple, S.L. BudIN, The Origin of Aphrodite, Bethesda, 2003; R. Rosenzweig, Worshipping Aphrodite: Art and Cult in Classical Athens, Ann Arbor, 2004; Ursula BITTRICH, Aphrodite und Eros in der antiken Tragödie, Berlin/New York, 2005 (pour un compte rendu de cet ouvrage, cf. supra, p. 412 sq.).

2 En préambule, je dois signaler que j'ai consacré une étude à la déesse et analysé, entre autres, son lien avec la guerre, dans une perspective différente de celle qu'a adoptée M. Valdès : cf. G. PIRONTI, Entre ciel et guerre. Figures d'Aphrodite en Grèce ancienne, Liège, 2007 (Kernos, suppl. 18). 
l'exclusion des femmes du pouvoir politique et de la guerre, comme une communauté d'hommes et de citoyens-guerriers.

En appliquant systématiquement ces hypothèses à la documentation, l'A. passe en revue les cultes d'Aphrodite dans la cité de Sparte (Chap. II), à Argos et en Arcadie (Chap. III), ensuite dans la cité de Corinthe (Chap. IV) et en Béotie (Chap. V), pour conclure avec Athènes (Chap. VI). Concernant, par exemple, l'Aphrodite armée de Sparte, qui était accompagnée, selon Pausanias, dans le même sanctuaire par une image de la déesse portant des chaînes, M.V. en conclut que ces représentations seraient " un signo o símbolo del sometimiento del peligro potencial de las mujeres especialmente en su faceta de reversión del orden establecido que podría tener su imagen en una Afrodita guerrera » (p. 50). Lorsqu'il s'agit d'expliquer les liens de l'Aphrodite argienne avec Arès et la sphère militaire, M.V. invoque le travestissement rituel accompagnant la fête des Hybristika ainsi que les récits locaux sur les exploits guerriers des femmes et la " gynécocratie-doulocratie », pour conclure que, pendant l'époque archaïque, le panthéon argien aurait connu Aphrodite « en su version de divinidad feminina guerrera ligada à la iniciación al matrimonio y a la guerra, al mismo tiempo que à la disolución ritual y momentánea del orden establecido non solo en relación con la mujeres, sino también con los esclavos » (p. 66). Dans l'effort de comprendre la façon dont la cité en formation aurait accueilli une divinité venue d'ailleurs, tout en l'adaptant à son propre système de valeurs, l'A. reprend donc l'interprétation de l'Aphrodite guerrière en termes de paradoxe, déjà adoptée par F. Graf.

Même s'il faut reconnaittre à M.V. d'avoir évité le piège d'une enquête centrée exclusivement sur les origines pour essayer de comprendre la fonction d'Aphrodite à l'intérieur même la société grecque, le parcours choisi et l'utilisation des sources soulèvent des questions d'ordre méthodologique. En l'état actuel de la documentation, il est impossible de faire remonter systématiquement à l'époque du haut archaïsme, ou de l'archaïsme tout court, les cultes d'Aphrodite analysés par l'A. Pausanias, qui est le témoin le plus souvent invoqué, ne suffit pas. De même, il apparait audacieux de dater l'introduction du culte d'Aphrodite à Athènes " entre el $1075 \mathrm{y}$ el 1025 » (p. 25) et d'appuyer cette hypothèse sur la tradition athénienne attribuant au roi Égée l'institution du culte de la déesse dans la cité. L'hypothèse de l'expansion en deux phases du culte d'Aphrodite se révèle également plutôt fragile, car elle se fonde moins sur de véritables indices que sur une série d'idées reçues concernant les origines de la déesse et le caractère à la fois « étrange » et « étranger» de ses traits guerriers.

Le parti pris selon lequel le lien d'Aphrodite avec la guerre serait plus accentué aux hautes époques pour s'estomper par la suite, est en effet contestable : d'un côté, l'association d'Aphrodite avec Arès parcourt toute l'Antiquité grecque, de l'autre, les prérogatives de la déesse dans la sphère politique et militaire sont bien attestées surtout aux époques classique et hellénistique. Certes, on ne peut exclure que cet aspect de la déesse remonte plus haut dans le temps, mais cette possibilité ne constitue pas une base assez solide pour fonder une théorie générale sur l'évolution de la personnalité d'Aphrodite et sa fonction dans la société grecque à l'époque archaïque. Cette approche fragilise d'autant plus la démonstration que l'A. ne résiste pas à la tentation d'ériger en certitudes des hypothèses privées de bases suffisamment solides. Ainsi, en recherchant à tout prix une Aphrodite guerrière dans l'Athènes archaïque, M.V. la retrouve dans une prétendue ancêtre d'Athéna Nikê, tout en n'ayant, pour asseoir cette hypothèse, que l'attribut de la grenade propre à cette Athéna et le nom d'une prêtresse dans la Lysistrata d'Aristophane (p. 105-110). Aucun culte d'une Aphrodite guerrière n'est attesté en Eubée à l'époque géométrique, mais une bague d'époque hellénistique provenant d'Érétrie et représentant une Aphrodite armée, conjointement à l'existence de contacts entre Chypre et l'Eubée à l'époque géométrique paraissent à l'A. des indices suffisants pour y croire. 
$\mathrm{Au}$ fond, la faiblesse de cette étude réside dans deux postulats qui sont étroitement liés l'un à l'autre: 1) pour comprendre la personnalité d'Aphrodite, il faut regarder vers les prétendues origines de cette déesse, donc vers Chypre et l'Orient; 2) l'implication de cette Aphrodite « hellénisée » dans l'univers guerrier ne peut s'expliquer qu'en termes de renversement de rôles, de travestissement rituel, de paradoxe. Or, en l'état actuel de la documentation, Chypre n'a livré que trop peu d'indices, et relativement récents, sur le culte local d'une Aphrodite liée à la guerre. Face au riche dossier qui nous vient des cités grecques, nous sommes dans l'obligation d'évaluer avant tout la possibilité que ce lien trouve sa raison d'être dans la pensée religieuse des Grecs eux-mêmes. Ensuite, la présence d'Aphrodite dans l'univers d'Arès, étant donné la variété chronologique et typologique des sources qui en témoignent, ne peut se ramener à une explication unique, mais demande à être analysée cas par cas en fonction du contexte : à ce propos, est-il pertinent de faire appel aux tensions entre hommes et femmes, ou même entre citoyens et esclaves, pour expliquer les armes d'Aphrodite? À mon sens, loin d'être paradoxal, l'aspect guerrier que cette déesse peut parfois revêtir engage à comprendre la façon dont elle est censée contribuer à la victoire militaire et à la protection de la cité. Certes, Aphrodite n'est pas Athéna, mais, comme les sources anciennes le confirment, les Grecs étaient bien conscients des recoupements entre guerre et sexualité.

Malgré ces limites, l'étude de M.V. se signale par une attention constante à l'imbrication profonde entre religion et société, et par une riche bibliographie, bien exploitée. Si la méthode suivie et les conclusions de cet ouvrage n'emportent pas la conviction, il faut toutefois reconnaître à M.V. le mérite d'avoir fait une part dans son enquête aux liens qu'Aphrodite entretient avec la guerre dans les cités grecques, un dossier souvent négligé et pourtant fondamental pour pouvoir cerner cette déesse dans toute sa complexité.

$$
\begin{array}{r}
\text { Gabriella Pironti } \\
\text { (Centre Louis Gernet, EHESS - Paris) }
\end{array}
$$

SAUZEAU Pierre, Les partages d'Argos. Sur les pas des Danaïdes, Paris, Belin, 2005. 1 vol. $14 \times 21 \mathrm{~cm}, 395$ p. (L'Antiquité au présent). ISBN : 2-7011-3346-7.

Das hier zu besprechende Buch von Pierre Sauzeau (Université de Montpellier III) ist seine für den Druck überarbeitete Dissertation von 1994 an der Universität Lyon II bei Pierre Vidal-Naquet und Michel Casevitz. Die Fragestellung beider Doktorväter begründen das Interesse einmal an sprachwissenschaftlichen Beobachtungen und an den Bildern (imaginaire), die sich mit der Stadt Argos verbinden.

Argos ist nach Athen und Sparta mit Korinth und Theben die bedeutendste Stadt Griechenlands, hat aber ungleich weniger Interesse und Forschungen gefunden. Und das, obwohl die großen Ausgrabungen der Amerikaner schon früh das Material des Heraions zur Verfügung gestellt haben und die französischen und griechischen Grabungen in der Stadt kontinuierlich (seit Willem Vollgraff) neues wichtiges Material zu Tage brachten. Die archäologischen Befunde sind in dem vorzüglichen Band Argos et Argolide. Topographie et Urbanisme (1998) zusammengefasst worden. Über das frühe Argos hat Robin Hägg wertvolle Beobachtungen gemacht, die er hoffentlich noch mit dem zweiten Band krönen wird. Und in der Zusammenschau von Archäologie und Texten hat Marcel Piérart fast Jahr um Jahr neue Erkenntnisse beigesteuert über Argos und seine Religionsgeschichte. Argos wird in den Arbeiten von de Polignac zum einen der beiden Idealtypen der Entstehung der griechischen Polis. Aber eine Monographie über Argos fehlt immer noch. So liest man mit Spannung dieses Werk.

Teil 1 will aus dem Namen Argos philologisch die Fülle der Möglichkeiten und die Eigenschaften erschließen, über die die Stadt verfügt. Angesichts der häufigeren Vorkom- 